\title{
E2F1-mediated repression of WNT5A expression Promotes Brain Metastasis Dependent on the ERK1/2 pathway in EGFR-Mutant Non-Small Cell Lung Cancer
}

Huanhuan Li ( $\nabla$ d201881451@hust.edu.cn )

Huazhong University of Science and Technology https://orcid.org/0000-0002-6513-6334

Fan Tong

Wuhan Union Hospital

Rui Meng

Wuhan Union Hospital

Ling Peng

Wuhan Union Hospital

Ruiguang Zhang

Wuhan Union Hospital

xiaorong Dong

Wuhan Union Hospital

\section{Research}

Keywords: WNT5A; EGFR mutation; NSCLC; E2F1; ERK1/2 signaling

Posted Date: May 22nd, 2020

DOI: https://doi.org/10.21203/rs.3.rs-30032/v1

License: (c) (1) This work is licensed under a Creative Commons Attribution 4.0 International License.

Read Full License

Version of Record: A version of this preprint was published at Cellular and Molecular Life Sciences on October 19th, 2020. See the published version at https://doi.org/10.1007/s00018-020-03678-6. 


\section{Abstract}

Background: Brain metastasis (BM) is associated with poor prognosis in patients with advanced nonsmall cell lung cancer (NSCLC). Epidermal growth factor receptor (EGFR) mutation reportedly enhances the development of BM. However, the exact mechanism of how EGFR-mutant NSCLC contributes to BM remains unknown. This study was aimed at exploring the mechanism of BM development in EGFRmutant NSCLC.

Methods: WNT5A, was identified by analyzing RNA sequencing data of BM tissue from NSCLC. The expression of WNT5A in NSCLC plasma $(n=94)$ and cells were detected by quantitative real-time PCR (qRT-PCR) and western blotting. WNT5A functions were examined by cell viability, migration, invasion, and immunohistochemistry assay in vitro. A xenograft nude mouse model and BM model were used to observe tumor growth and brain metastasis in vivo. The potential transcription factor of WNT5A was explored using bioinformatics analysis and verified by chromatin immunoprecipitation (ChIP) and luciferase reporter assays. WNT5A targets in NSCLC cells were confirmed using luciferase reporter assay, qRT-PCR, and western blotting.

Results: WNT5A was downregulated in BM tissues and EGFR-mutant samples and cells. The overexpression of WNT5A inhibited the growth, migration, and invasion of EGFR-mutant cells in vitro and retarded tumor growth and metastasis in vivo compared to the EGFR wide-type cells. ChIP and luciferase reporter assays showed that E2F1 negatively regulated WNT5A at transcriptional levels, which was suppressed by ERK1/2 inhibitor (U0126) in EGFR-mutant cells. Furthermore, WNT5A inhibited $\beta$-catenin activity and the transcriptional levels of its downstream genes in cancer progression.

Conclusions: Our research revealed the role of WNT5A in NSCLC BM with EGFR mutation and proved that E2F1-mediated repression of WNT5A was dependent on the ERK1/2 pathway, supporting the notion that targeting the ERK1/2-E2F1-WNT5A pathway could be an effective strategy for treating BM in EGFRmutant NSCLC.

\section{Introduction}

Lung cancer is the most commonly diagnosed cancer and the leading cause of cancer death worldwide ${ }^{[1]}$. Non-small cell lung carcinoma (NSCLC) constitutes approximately $85 \%$ of all lung cancer cases $^{[2]}$ and is frequently characterized by the presence of brain metastasis (BM), which is common in patients with advanced NSCLC with the incidence rate of more than $25 \%{ }^{[3]}$. BM is associated with poor overall survival (OS) and causes significant peripheral neuropathy, cognitive effects, and emotional consequences $^{[4]}$.

Epidermal growth factor receptor (EGFR) mutations are observed in approximately $50 \%$ of patients with lung cancer ${ }^{[5]}$. EGFR mutation induces ligand-independent receptor dimerization and activation, resulting in the phosphorylation of EGFR and activation of the downstream signaling cascade, such as the 
$\mathrm{PI3K} / \mathrm{AKT}$ or extracellular signal-regulated kinase 1/2 (ERK1/2) pathway ${ }^{[6,7]}$. Emerging evidence suggests that EGFR-mutant advanced NSCLC is particularly prone to develop the BM, implying that EGFR mutation could be a risk factor for BM in NSCLC ${ }^{[5,8]}$. However, the exact mechanism of how EGFR mutation in lung cancer contributes to brain metastasis remains largely unknown.

Unlike canonical Wnt/ $\beta$-catenin signaling, WNT5A, as a member of non-canonical Wnt signaling, controls various aspects of cell migration, which are essential in normal and malignant development ${ }^{[9,10]}$ and the aberrant expression of WNT5A induces distinct effects in different types of cancer ${ }^{[10-16]}$. Previous studies have shown that WNT5A has two protein isoforms, namely WNT5A-long and WNT5A-short; the former inhibits the proliferation of tumor cell lines, while the latter promotes their growth ${ }^{[17]}$. In addition, WNT5A has a vital role in neuron development in association with the EGFR pathway ${ }^{[18]}$. However, the underlying mechanism for this correlation in BM with lung cancer has not yet been elucidated.

In this study, we revealed the antitumor effects of WNT5A in EGFR-mutant NSCLC. And identified ERK1/2E2F1-WNT5A pathway which may be critical for progression of BM in EGFR-mutant NSCLC.

\section{Methods}

\section{Patients samples}

Peripheral blood samples were collected from 94 NSCLC patients enrolled in the Union Hospital of Tongji Medical College, HUST. The demographic and clinicopathological details of patients showed in Additional file 1: Table S1. Among the cases, 68 were sequenced by Next-generation sequencing (NGS) to identify the mutation status of EGFR, including $35 \mathrm{BM}+$ and $33 \mathrm{BM}$ - The Institutional Review Board of Huazhong University of Science and Technology approved this study. Written informed consent was obtained from all legal guardians of the patients.

\section{Cell lines}

A549 and HCC827 cell lines were acquired from the Institute of Biochemistry and Cell Biology of the Chinese Academy of Sciences, Shanghai, China. H1299, H1975, and 293T cell lines were procured from the American Type Culture Collection (ATCC). HCC827 cells harbored an activating in-frame deletion in exon 19 (Ex19del) of EGFR, whereas H1975 cells harbored activating L858R and T790M mutations in exon 21 of EGFR. A549 and H1299 were EGFR wide-type (wt) cells. All cells were cultured in an RPMI 1640 medium (Gibico, Grand Island, NY, USA) supplemented with $10 \%$ fetal bovine serum (10\% FBS). Cells were grown at $37{ }^{\circ} \mathrm{C}$ in a humidified atmosphere of $5 \% \mathrm{CO}_{2}$. The cell identification report is shown in the attached data.

\section{Real-time quantitative RT-PCR (qRT-PCR) and PCR.}

Total RNA was extracted from plasma samples using a BIOG RNA IsoQuick Kit (\#61011), and the RNA from cells was isolated using a TRIzol reagent (Invitrogen) and then subjected to reverse transcription 
with the PrimeScript RT Reagent Kit (Takara, China).The qRT-PCR was performed by means of the SYBR Premix Ex Taq II kit (Takara, China). The data were analyzed using the $2^{-\triangle \Delta C T}$ method. The primer design and sequence are shown in Additional file 1: Table S2.

\section{Overexpression and knockdown of genes}

Human E2F1 overexpression vector and shRNA were purchased from GenePharma (Shanghai, China). An empty vector (Mock) and scramble shRNA (sh-Scb) were used as controls. Lentiviral WNT5A overexpression and the empty vector, which carry the EF1 promoter-driven firefly luciferase and puromycin resistance gene, and the corresponding virus were purchased from GenePharma (Shanghai, China).

\section{Establishment of stable lung cancer cell lines}

WNT5A viral vectors were transfected into HCC827 and H1299 cells according to the operating manual, and after screening with puromycin (Invitrogen), stable cancer cells were established. The E2F1 overexpression vector and shRNA were also transfected, and selected with puromycin $(5 \mu \mathrm{g} / \mathrm{ml})$, stable cell lines HCC827 and H1975 were obtained.

\section{Drugs}

Icotinib (99.9\% purity) was kindly supplied by Zhejiang Beta Pharmalnc (Zhejiang, China) and dissolved in dimethyl sulfoxide (DMSO, Sigma, St. Louis, MO, USA) to $50 \mathrm{mM}$ of stock solution and stored at $-20^{\circ} \mathrm{C}$ and then diluted with culture medium before use.

\section{Cell counting Kit-8 assay}

The cell viability assay was conducted following the instructions for the use of the CCK-8 kit (Dojindo Laboratories, Kumamoto, Japan). Tumor cells $\left(5 \times 10^{3}\right.$ cells/well) were seeded in 96 -well plates, and cell viability was determined by measuring the absorbance at $450 \mathrm{~nm}$. All experiments were replicated three times.

\section{Western blotting}

Tumor cell or tissue protein was prepared using $1 \times$ cell lysis RIPA buffer (Beyotime) supplemented with a protease inhibitor cocktail (Thermo Scientific, Waltham, MA, USA) and PMSF. Western blotting was performed as described previously ${ }^{[19,20]}$. Anti-EGFR (\#4267), anti-pEGFR (\#3777), anti-AKT (\#4691), antipAKT (\#4060), anti-ERK1/2 (\#4695), anti-pERK1/2 (\#4370), and anti-E2F1 (\#3742) antibodies were purchased from Cell Signaling Technology (CST, Danvers, MA). Anti-WNT5A (A12744), anti-b-catenin (A11512), and anti-GAPDH (AC033) were acquired from ABclonal (USA), and anti-Histone H3 (ab176842) was procured from Abcam (USA)

\section{Chromatin immunoprecipitation (ChIP)}


ChIP assay was carried out using the ChIP-IT \& Express kit (Cat. No. 53008; Active Motif, Carlsbad, CA, USA) following the manufacturer's protocol. Antibodies used for this assay included control IgG (ab150081, Abcam) and antibody specific to E2F1 (\#3742, CST). DNA was extracted and sonicated into a 200 bp size. For qRT-PCR, the gene promoter-specific primers (human WNT5A promoter bearing E2F1 site [-823/-806]) (Figure S3A) were used, as explicitly described in a previous report ${ }^{[21]}$.

\section{Luciferase reporter assay}

TOP-FLASH and FOP-FLASH reporters were obtained from Millipore. A human WNT5A luciferase reporter was established by inserting oligonucleotides containing E2F1 binding sites into pGL3-Basic (TsingKE biological technology). The pRL-TK vector was used as an internal control for luciferase activity (Promega). Relative $\beta$-catenin activation was determined using the TOP-FLASH/FOP-FLASH ratio. Dualluciferase assay was undertaken, as reported previously[22].

\section{Transwell invasion assays}

The invasion assays was conducted in transwell chambers (Costar, Corning Inc., NY, USA), starved tumor cells $\left(1.5 \times 10^{6}\right)$ were transferred onto the upper chamber coated with Matrigel (BD Biosciences, San Jose, CA, USA). After $24 \mathrm{~h}$ of incubation, invaded cells were stained with $0.1 \%$ crystal violet (Sigma). Counted under the microscope.

(Olympus, Tokyo, Japan).

\section{Wound-healing assay}

For the wound-healing assay, cells were seeded into 6 -well plates $\left(1 \times 10^{5}\right.$ cells/well), and the monolayer was scratched with a $10 \mu \mathrm{l}$ plastic pipette tip to create a uniform wound. The wound margin distance between the two edges of the migrating cell sheets was photographed under a phase-contrast microscope. All experiments were performed in triplicates.

\section{In vivo xenograft assay}

Tumor cells were prepared by suspending $6 \times 10^{6} \mathrm{HCC} 827$ and $\mathrm{H} 1299$ cells in $100 \mu$ of serum-free media and injecting into the right rear flanks of 4-weeks-old female BALB/c nude mice (Beijing Huafukang Bioscience Company, Beijing, China). Tumor growth was monitored and recorded every week after the inoculation. Tumor volume was calculated as follows: $0.5 \times$ tumor length $\times$ tumor width ${ }^{2}$. Four weeks later, tumor burden were evaluated and tumors were excised and dissected for characterization and further studies. All animal experiments were performed under the protocol approved by the Institutional Animal Care and Use Committee of Huazhong University of Science and Technology.

\section{Brain metastatic xenografts}


The mouse model of brain metastasis was performed as described previously ${ }^{[20]}$. Female nude mice $(5 \sim$ 6 weeks of age) were purchased from Beijing Hua Fukang Bioscience Company (Beijing, China). H1299 and HCC827 cell suspensions were prepared by stably transfecting the cells with WNT5A lentivirus $\left(1 \times 10^{7}\right.$ cells $/ \mathrm{mL}$ in PBS). $50 \mu \mathrm{L}$ of cell suspension was slowly injected into the intracarotid artery of nude mice. The In Vivo Optical Imaging System (In Vivo FX PRO, Bruker Corporation) was used to acquire fluorescent images of each mouse after an intraperitoneal injection of $150 \mathrm{mg} / \mathrm{kg}$ luciferin at a final concentration of $15 \mathrm{mg} / \mathrm{mL}$ (Goldbio St. Loius, MO, USA). The presence of brain metastases was histopathologically confirmed.

\section{Immunohistochemical (IHC) analysis}

Immunohistochemical staining and quantitative evaluations were performed as previously described ${ }^{[23]}$. Antibodies used included Ki-67 (7309-1-AP, Proteintech) and CD31 (ab28364, Abcam). At least two pathologists blinded to the investigation assessed the degree of positivity. The IHC scoring system consisted of determining the percentage of positively stained tumor cells and estimating the intensity of staining. The percentage of Ki-67 positive cells was expressed as the ratio between the number of positive tumor cells and the total number of tumor cells counted in the field. The mean number of microvessels in the five parenchymal areas detected by CD31 in tumor areas was regarded as the microvessel density (MVD).

\section{Data mining of public datasets}

The RNA sequencing datasets of NSCLC patients with or without BM had been deposited in Gene Expression Omnibus (GEO) and were accessible with the login number GSE126548. The differentiallyexpressed genes identified by RNA Sequencing from GEO datasets GSE16476 were subjected to oncogenic signature enrichment analysis. Public GEO datasets GSE14340 were established using the Affymetrix Human Genome U133 Plus 2.0 Array platform (GPL570). (Genes upregulated in MCF-7 cells (breast cancer) positive for ESR1 [GenelD=2099] and engineered to express ligand-activatable EGFR) was accessed to find genes rich in EGPR signature. Differentially expressed genes between two groups were screened using unpaired Student's t-test and correction multiple testing methods. The log-rank test and correction multiple testing analyses were applied to evaluate the survival significance of each gene in NSCLC patients.

\section{Statistical analysis}

Data from the GEO database (GSE126548) were processed as aforementioned. Results were reported as the mean \pm SEM. The cutoff for gene expression was defined by average values. Comparisons between two groups were made using the unpaired t-test. The differences between multiple groups were determined using the one-way ANOVA and the posthoc Tukey HSD test. The statistical significance of overlap or the expression of correlation $\mathrm{n}$ was determined using Fisher's exact test and Pearson's productmoment correlation analysis, respectively. The log-rank assay was employed to compare the difference in survival. $P<0.05$ was considered to be statistically significant. 


\section{Results}

\section{WNT5A expression decreased in patients with brain metastasis of NSCLC}

To unearth the genes crucial for BM of NSCLC, we performed RNA sequencing analysis to screen the differentially expressed genes in primary tissues of NSCLC patients with BM+ and BM- (GSE126548). As shown in Fig 1A, the EGFR signature was enriched according to the molecular signatures Database $(\mathrm{p}<$ 0.05 ) according to oncogenic signature enrichment analysis. Furthermore, the 182 genes rich in the EGPR signature were analyzed by accessing Gene Set EGFR_UP.V1_UP. As shown in Fig 1B-C, the heatmap and volcano plot showed that WNT5A expression was significantly downregulated in the BM+ group, suggesting that WNT5A expression has some potential relevance to BM in NSCLC.

\section{Loss of WNT5A expression was associated with a poor clinical outcome in NSCLC}

Analysis of the Kaplan-Meier plotter ${ }^{[24]}$ revealed that the high expression of WNT5A in NSCLC patients was associated with a good outcome (Hazard ratio $[\mathrm{HR}]=0.83, p=0.003$ ) (Fig 1D), a HR below 1 suggests a smaller risk. In addition, WNT5A expression was lower in lung tumor tissues of BM+ NSCLC patients compared to the BM- group ( $n=5)$, by employing IHC assay (Fig 1E). Moreover, 94 plasma cases of NSCLC patients were evaluated by qRT-PCR, and showed that WNT5A expression in BM+ NSCLC patients $(n=48)$ was significantly lower compared with the BM- group $(n=46)(P=0.0478)(F i g 1 F)$. Furthermore, we screened the BM+ NSCLC that had been analyzed by NGS $(n=35)$. Notably, the expression of WNT5A in the EGFR-mutant group ( $n=25)$ was significantly lower than that of the EGFR wild type (wt) group ( $n=10)$ (Fig 1G) $(P=0.0262)$. We also found that EGFR mutation was more inclined to occur in $\mathrm{BM}$, which was in consistent with the previous reports (Additional file 1: Table S1). These results also suggest that the loss of WNT5A might be involved in the development of BM in EGFR-mutant NSCLC.

\section{WNT5A inhibits the growth and aggressiveness of EGFR-mutant NSCLC cells}

To confirm the hypothesis that WNT5A was a key factor in EGFR-mutant BM in NSCLC in vitro, we explored the expression of WNT5A on EGFR-mutant or wt cell lines. Compared with EGFR wt cell lines (A549 and H1299), the expression of WNT5A was lower in EGFR-mutant cell lines (HCC827 and H1975), with an increase in the accompanying basal level of EGFR phosphorylation (p-EGFR) expression (Fig 2A). Furthermore, icotinib $(0.1 \mu \mathrm{M}, 24 \mathrm{~h})$, inhibit EGFR activation, resulted in the upregulation of WNT5A expression in HCC827, but on the contrary, no significant changes in the H1299 (Fig 2B). These data suggest that the expression of WNT5A is dependent on EGFR-mutant-induced activation signaling in NSCLC.

To explored the role of WNT5A in EGFR-mutant NSCLC, the H1299 and HCC827 cells stable transfection of WNT5A lentivirus were constructed (Additional file 2: Fig S1A-B). Remarkably, WNT5A transfected EGFR-mutant cell lines exhibited more apparent inhibition ability of cell proliferation per the CCK-8 assay (Fig 2C). Also, wound healing assay and matrigel invasion assays revealed that WNT5A more 
significantly inhibited the migration and invasiveness of EGFR-mutant cells in comparison with EGFR wt cells (Fig 2D-F). These results suggest that WNT5A suppresses cell growth and aggressiveness, specifically in EGFR-mutant NSCLC cells in vitro.

\section{WNT5A suppressed tumorigenesis and brain metastasis in vivo}

To further elucidate the anti-tumor effect of WNT5A in EGFR-mutant NSCLC cells in vivo, the xenograft model and BM model of mice were established by implanting stably-transfected HCC827 and H1299 cells with WNT5A overexpression. The overexpression of WNT5A significantly inhibited tumor growth and tumor size in HCC827 group. In contrast, the inhibitory effect in the H1299 group was slight (Fig 3A-C and Additional file 2: Fig S1C-D). In addition, a higher survival probability was noted in nude mice treated with HCC827 cells stably overexpressing WNT5A, but not evident in the H1299 group ( $P<0.05)$ (Fig 3D). Moreover, $\mathrm{IHC}$ staining showed that the expression of $\mathrm{Ki}-67$ and $\mathrm{CD} 31$ diminished significantly in cells overexpressing WNT5A $(\mathrm{P}<0.05)($ Fig 3E-F).

Consistently, in the BM model of mice, the overexpression of WNT5A significantly inhibited the occurrence of BM in the EGFR-mutant group $(P<0.05)$ (Fig 4A-B). A greater survival probability of mice was noted by stably overexpressing WNT5A in the HCC827 group, compared with the H1299 group (Fig 4C). Additionally, IHC staining indicated that the expression of Ki-67 and CD31 diminished significantly with WNT5A overexpressed, especially in the HCC827 group $(P<0.05)$ (Fig 4D-E). All these findings show that WNT5A decreased the growth and aggressiveness of EGFR-mutant cell lines and is a potential therapeutic target in BM of EGFR-mutant NSCLC cells in vivo.

\section{E2F1 regulated the repression of WNT5A in EGFR-mutant NSCLC}

To investigate the underlying mechanism of WNT5A loss following EGFR mutation signaling in NSCLC, we analyzed publicly available datasets UCSC Genome Browser and Genomatix to filter any transcriptional factors (TFs) (Additional file 1: Table S3) that may regulate WNT5A expression. Based on the overlapping analyses of these genes, five potential TFs, namely E2F1, AP-1 transcription factor subunit (AP-1), NF-KB, paired box 2 (PAX2), and POU class 2 homeobox 1 (POU2F1) were identified (Fig $5 A)$. According to a $p$-value and HR value, E2F1 was the only TF considered, the high expression of E2F1 was associated with poor outcomes in NSCLC $(H R=1.46, p=5 E-09)$, and correlating negatively with the expression of WNT5A $(R=-0.28, P<0.01)$ (Fig 5B-C). The other 4 TFs were AP-1 $(H R=1.13, p=2.6 E-05)$, NF-KB $(H R=0.91, p=0.15)$, PAX2 (HR = 0.75, $p=0.056)$, and POU2F1 (HR = 0.79, $p=9.3 E-05)$ (Additional file 3: Fig S2A-B). Accordingly, these data suggest that E2F1 has the potential ability to suppress WNT5A expression at the transcriptional level in NSCLC.

To explore the impact of E2F1 on the expression of WNT5A in EGFR-mutant NSCLC, the overexpression and knockdown vector of E2F1 were transfected into HCC827 and $\mathrm{H} 1975$ cells, respectively (Additional file 3: Fig S2C-D). Notably, the mRNA levels of WNT5A decreased significantly in NSCLC cells overexpressing E2F1, whereas, knocking down E2F1 produced the opposite effect (Fig 5D), indicating that E2F1 regulated the repression of WNT5A in EGFR-mutant NSCLC cells. 
Subsequently, to verify E2F1 acts as a transcriptional regulator of WNT5A, we analyzed the potential binding motif of the WNT5A promoter region by mining public databases UCSC Genome Browser and JASPAR, and unearthed two putative E2F1-binding sites with the consensus sequence of GGCGCCAAA (Fig 5E and Additional file 4: Fig S3A). Considering the WNT5A-long isoform exerts anti-tumor effects, site1 was chosen as the potential binding site. ChIP assays were performed with E2F1 antibodies and revealed that endogenous E2F1 recruitment to the promoter of WNT5A, and the binding ability was mainly reflected in the HCC827 group compared with the H1299 group (Fig 5F and Additional file 4: Fig S3B). In addition, the rescue experiments indicated that the ectopic expression of E2F1 abolished the change in the promoter enrichment of WNT5A induced by treatment with icotinib in EGFR-mutant NSCLC cells (Fig 5G-H).

Furthermore, HEK293T cells transfected with E2F1 were subjected to luciferase reporter assays, revealed that the ectopic expression of E2F1 suppressed the transcriptional activation of WNT5A, whereas shE2F1 facilitated such a ability (Additional file 4: Fig S3C). The ectopic expression of E2F1 also prevented increased transcriptional activation of WNT5A induced by icotinib in HCC827 and H1975 cells (Fig 5I). Additionally, the overexpression of E2F1 suppressed the transcriptional and protein levels of WNT5A in EGFR-mutant cells and reversed the increased WNT5A expression induced by icotinib (Fig 6A-B). Overall, findings suggest that E2F1 binds to the promoter of WNT5A directly and suppresses the expression of WNT5A at transcriptional and protein levels in EGFR-mutant cells.

\section{The E2F1/WNT5A axis downregulates $\beta$-catenin activity mediated by the ERK1/2 pathway in EGFR- mutant cells}

WNT5A has been reported to inhibit canonical Wnt/ $\beta$-catenin signaling ${ }^{[25]}$, to determine whether $\beta$-catenin signaling is a potential pathway for WNT5A mediation of BM in NSCLC, the expression and activity of $\beta$ catenin were measured in EGFR-mutant cells. Indeed, WNT5A did not inhibit the levels of $\beta$-catenin efficiently but subdued the transcription levels of its downstream target genes, including CCDN1 (cyclin D1), CD44 (CD44 molecule), and ZEB1 (zinc finger E-box binding homeobox 1) significantly (Fig 6C-D and Additional file 4: Figure S3D). Furthermore, the TOP/FOP flash assay showed that WNT5A attenuated $\beta$ catenin activity in EGFR mutant cells significantly (Fig 6E).

ERK1/2 and PI3K/AKT singling are classic pathways that mediate EGFR signals ${ }^{[26]}$. Furthermore, U0126 (a MEK inhibitor) inactivated ERK1/2 singling and suppressed the E2F1-mediated repression of WNT5A in EGFR-mutant cells (Fig 6F). However, LY294002 (a PI3K inhibitor) inhibited the AKT pathway but no significant effect on the expression of the E2F1/WNT5A axis (Additional file 4: Fig S3E). These data indicated EGFR mutation occurs via ERK1/2 signaling, instead of the PI3K/AKT pathway, to enhance E2F1 binding to the WNT5A promoter and repress its activity, thereby increasing $\beta$-catenin activity (Fig 6G-H and Additional file 4: Fig S3F-G). Collectively, the data suggest that E2F1 downregulates WNT5A expression at transcriptional levels and relieves the inhibition of $\beta$-catenin, which in turn upregulating $\beta$ catenin-induced reporter gene expression and promoting EGFR-mutant-induced BM in NSCLC, and these processes mainly depend on the ERK $1 / 2$ pathway(Fig 6I). 


\section{Discussion}

BM is one of the major causes of death in advanced NSCLC and is associated with short survival and reduced quality of life $\mathrm{e}^{[27,28]}$. The EGFR mutation status is a predictive factor response to EGFR-TKIs, which could significantly prolong the progression-free survival (PFS) and objective response rate (ORR) of NSCLC patients with $\mathrm{BM}^{[28,29]}$. Accumulating evidence shows that EGFR-mutant NSCLC patients are more prone to develop $\mathrm{BM}^{[30-32]}$. However, the underlying mechanisms of how EGFR mutation contributes to BM in NSCLC remain largely unknown. In this study, we demonstrate that the E2F1/WNT5A axis is a novel mechanism involved in the occurrence of BM in EGFR-mutant NSCLC. Our initial evidence reveals that overexpression of WNT5A is associated with less BM in EGFR-mutant NSCLC and better outcome of patients. Our findings indicate that the WNT5A is negatively regulated by E2F1 at transcription levels and the process was dependent on the ERK1/2 pathway. The activation of ERK1/2-E2F1-WNT5A pathway contributes to the occurrence of BM in EGFR-mutant NSCLC. These outcomes represent a promising step in the diagnosis and treatment of EGFR-mutant NSCLC.

WNT5A belongs to Wnt family, exerting a critical role in the regulatory processes of an organism and performing anti- or pro-tumorigenic roles in cancer progression ${ }^{[11,33]}$. The aberrant expression of WNT5A is reportedly involved in the progression of cancer ${ }^{[16,34,35]}$. In this study, we identified WNT5A exerts tumor-suppressive functions, inhibiting the growth and aggressiveness of EGFR-mutant NSCLC cells, via influencing the activity of $\beta$-catenin and downregulating the downstream gene expression, including CCND1, CD44 and ZEB1. Notably, our findings indicated that the other WNT5A variants, WNT5A-short ${ }^{[17]}$, might exert opposite oncogenic functions in tumorigenesis and aggressiveness, which needed further investigation.

Multiple studies have highlighted the critical role of transcription regulation in the progression of cancers $^{[36,37]}$. E2F1 was reported mediating transcriptional activation or inhibition in a variety of tumors, such as downregulating the expression of the tumor suppressor gene ARHI in breast cancer cells via complexing with histone deacetylase (HDAC) and enhancing the metastasis of tumor cells by upregulating vascular endothelial growth factor ${ }^{[38-40]}$. E2F1 also promotes the invasion of NSCLC and is associated with poor prognosis in NSCLC ${ }^{[41]}$. Moreover, E2F1 is required for ligand-independent EGFR activation and promotes gut regeneration via EGFR/MAPK signaling ${ }^{[42]}$. In our study, we showed that E2F1 decreased the WNT5A activity by binding to its promoter, resulting in the transcriptional repression of WNT5A. It has been indicated that WNT5A promoter being epigenetically activated during transformation in Glioblastoma ${ }^{[43]}$. Whether epigenetic modification also involved in the regulation of WNT5A promoter by E2F1, warrants further investigation.

The PI3K/AKT and ERK1/2 signaling pathways are constitutively activated in NSCLC tissues with EGFR mutation compared to EGFR wt ${ }^{[44,45]}$. The aberrant activation of the complex intracellular signaling pathways have been causally linked to cancers, inflammation, and angiogenesis ${ }^{[26]}$. In the current study, we identified that E2F1-mediated suppression of WNT5A in EGFR-mutant NSCLC was mainly regulated by 
the ERK1/2, rather than the PI3K/AKT pathway. There is possibility that other signaling pathways are also involved, and the full mechanisms of our outcomes remain to be elucidated in a future study. Previous studies have shown that the activation of EGFR signaling inactivated Rb, leading to the release of E2F1 from its sequestration to perform transcriptional regulatory functions ${ }^{[46]}$, which may be the potential mechanism of E2F1 plays a role in EGFR-mutant NSCLC, worthing further exploration. our evidence indicates that the E2F1-WNT5A axis in EGFR-mutant NSCLC, at least in part, mediated by ERK1/2 pathway.

\section{Conclusion}

In summary, our study shows that WNT5A inhibited EGFR-mutant cell proliferation, metastasis, and tumorigenesis both in vitro and in vivo. The suppression of WNT5A was regulated by E2F1 and the E2F1/WNT5A axis was mediated in part via the ERK1/2 pathway in EGFR-mutant NSCLC. These findings have significant implications for the understanding of EGFR-mutant more likely to have BM in NSCLC, and offers a new possible therapeutic strategy for NSCLC.

\section{Abbreviations}

BM: Brain metastasis; NSCLC: Non-small cell lung cancer; EGFR: Epidermal growth factor receptor; TKI: tyrosine kinase inhibitor; RK1/2: Extracellular signal-regulated kinase 1/2; $\beta$-catenin: catenin beta 1; E2F1: E2F transcription factor 1; PI3K: Phosphoinositide 3-kinase; HDAC: histone deacetylase; cDNA: Complementary DNA; qRT-PCR: quantitative real-time PCR; ChIP: Chromatin immunoprecipitation; HR: hazard ratio; mut: mutation; wt: Wild-type; GEO: Gene Expression Omnibus; TFs: transcriptional factors; OS: overall survival

\section{Declarations}

\section{Declaration of Competing Interest}

The authors declare no conflicts of interest.

\section{Acknowledgments}

Not applicable.

\section{Authors' contributions}

XRD conceived the study. HHL, LP, and JJW performed the experiments. HHL, FT and RM analyzed the data. XRD and HHL wrote the manuscript. All authors read and approved the final manuscript.

\section{Ethics approval and consent to participate}


This study was approved by the Institutional Review Board of Huazhong University of Science and Technology. Written informed consent was obtained from all legal guardians of the patients. All animal experiments were conducted in agreement with the Guide for the Care and Use of Laboratory Animals and were approved by the Committee on Animal Handling of Huazhong University of Science and Technology.

\section{Funding}

This work was supported by the National Natural Science Foundation of China $(81573090,81172595)$.

\section{References}

[1] Bray F, Ferlay J, Soerjomataram I, Siegel RL, Torre LA, Jemal A. Global cancer statistics 2018: Globocan estimates of incidence and mortality worldwide for 36 cancers in 185 countries. CA: a cancer journal for clinicians. 2018;68(6):394-424.

[2] Siegel RL, Miller KD, Jemal A. Cancer statistics, 2017. CA: a cancer journal for clinicians. 2017;67(1):7-30.

[3] Langer CJ, Mehta MP. Current management of brain metastases, with a focus on systemic options. Journal of clinical oncology : official journal of the American Society of Clinical Oncology. 2005;23(25):6207-6219.

[4] Yang J, Gong W. Lorlatinib for the treatment of anaplastic lymphoma kinase-positive non-small cell lung cancer. Expert review of clinical pharmacology. 2019;12(3):1-6.

[5] Yang Z, Guo Q, Wang Y, Chen K, Zhang L, Cheng Z, et al. Azd3759, a bbb-penetrating egfr inhibitor for the treatment of egfr mutant nsclc with cns metastases. Science translational medicine. 2016;8(368):368ra172.

[6] Lorimer IA, Lavictoire SJ. Activation of extracellular-regulated kinases by normal and mutant egf receptors. Biochimica et biophysica acta. 2001;1538(1):1-9.

[7] Moscatello DK, Holgado-Madruga M, Emlet DR, Montgomery RB, Wong AJ. Constitutive activation of phosphatidylinositol 3-kinase by a naturally occurring mutant epidermal growth factor receptor. The Journal of biological chemistry. 1998;273(1):200-206.

[8] Tan L, Wu Y, Ma X, Yan Y, Shao S, Liu J, et al. A comprehensive meta-analysis of association between egfr mutation status and brain metastases in nsclc. 2019;25(2):791-799.

[9] Famili F, Naber BA, Vloemans S, de Haas EF, Tiemessen MM, Staal FJ. Discrete roles of canonical and non-canonical wnt signaling in hematopoiesis and lymphopoiesis. Cell death \& disease. 2015;6:e1981. 
[10] van Amerongen R. Alternative wnt pathways and receptors. Cold Spring Harbor perspectives in biology. 2012;4:10.

[11] Asem MS, Buechler S, Wates RB, Miller DL, Stack MS. Wnt5a signaling in cancer. Cancers. 2016;8:9.

[12] Kremenevskaja N, von Wasielewski R, Rao AS, Schofl C, Andersson T, Brabant G. Wnt-5a has tumor suppressor activity in thyroid carcinoma. Oncogene. 2005;24(13):2144-2154.

[13] Roman-Gomez J, Jimenez-Velasco A, Cordeu L, Vilas-Zornoza A, San Jose-Eneriz E, Garate L, et al. Wnt5a, a putative tumour suppressor of lymphoid malignancies, is inactivated by aberrant methylation in acute lymphoblastic leukaemia. European journal of cancer. 2007;43(18):2736-2746.

[14] Borcherding N, Kusner D, Kolb R, Xie Q, Li W, Yuan F, et al. Paracrine wnt5a signaling inhibits expansion of tumor-initiating cells. Cancer research. 2015;75(10):1972-1982.

[15] Gujral TS, Chan M, Peshkin L, Sorger PK, Kirschner MW, MacBeath G. A noncanonical frizzled2 pathway regulates epithelial-mesenchymal transition and metastasis. Cell. 2014;159(4):844-856.

[16] Prasad CP, Manchanda M, Mohapatra P, Andersson T. Wnt5a as a therapeutic target in breast cancer. Cancer metastasis reviews. 2018;37(4):767-778.

[17] Bauer M, Benard J, Gaasterland T, Willert K, Cappellen D. Wnt5a encodes two isoforms with distinct functions in cancers. PloS one. 2013;8(11):e80526.

[18] Yoon S, Choi MH, Chang MS, Baik JH. Wnt5a-dopamine d2 receptor interactions regulate dopamine neuron development via extracellular signal-regulated kinase (erk) activation. The Journal of biological chemistry. 2011;286(18):15641-15651.

[19] Wei C, Dong X, Lu H, Tong F, Chen L, Zhang R, et al. Lpcat1 promotes brain metastasis of lung adenocarcinoma by up-regulating pi3k/akt/myc pathway. Journal of experimental \& clinical cancer research : CR. 2019;38(1):95.

[20] Wei CH, Wu G, Cai Q, Gao XC, Tong F, Zhou R, et al. Microrna-330-3p promotes cell invasion and metastasis in non-small cell lung cancer through gria3 by activating mapk/erk signaling pathway. Journal of hematology \& oncology. 2017;10(1):125.

[21] Li D, Song H, Mei H, Fang E, Wang X, Yang F, et al. Armadillo repeat containing 12 promotes neuroblastoma progression through interaction with retinoblastoma binding protein 4 . Nature communications. 2018;9(1):2829.

[22] Li D, Chen $Y$, Mei H, Jiao W, Song $H$, Ye L, et al. Ets-1 promoter-associated noncoding rna regulates the nono/erg/ets-1 axis to drive gastric cancer progression. Oncogene. 2018;37(35):4871-4886. 
[23] Li H, Yang F, Hu A, Wang X, Fang E, Chen Y, et al. Therapeutic targeting of circ-cux $1 /$ ewsr1/maz axis inhibits glycolysis and neuroblastoma progression. EMBO Molecular Medicine. 2019;11(12):e10835.

[24] Nagy A, Lanczky A, Menyhart O, Gyorffy B. Validation of mirna prognostic power in hepatocellular carcinoma using expression data of independent datasets. Scientific reports. 2018;8(1):9227.

[25] Mikels AJ, Nusse R. Purified wnt5a protein activates or inhibits beta-catenin-tcf signaling depending on receptor context. PLoS biology. 2006;4(4):e115.

[26] Lemmon MA, Schlessinger J. Cell signaling by receptor tyrosine kinases. Cell. 2010;141(7):11171134.

[27] Remon J, Ahn MJ, Girard N, Johnson M, Kim DW, Lopes G, et al. Advanced stage non-small cell lung cancer: Advances in thoracic oncology 2018. Journal of thoracic oncology : official publication of the International Association for the Study of Lung Cancer. 2019;14:7.

[28] Ponce S, Bruna J, Juan O, Lopez R, Navarro A, Ortega AL, et al. Multidisciplinary expert opinion on the treatment consensus for patients with egfr mutated nsclc with brain metastases. Critical reviews in oncology/hematology. 2019;138(190-206):190-206.

[29] Lee Y, Kim TM, Kim DW, Kim S, Kim M, Keam B, et al. Pre-clinical modeling of osimertinib for nonsmall cell lung cancer with egfr exon 20 insertion mutations. Journal of thoracic oncology : official publication of the International Association for the Study of Lung Cancer. 2019; pii: S1556-0864(19).

[30] Ge M, Zhuang Y, Zhou X, Huang R, Liang X, Zhan Q. High probability and frequency of egfr mutations in non-small cell lung cancer with brain metastases. Journal of neuro-oncology. 2017;135(2):413-418.

[31] Hendriks LE, Smit EF, Vosse BA, Mellema WW, Heideman DA, Bootsma GP, et al. Egfr mutated nonsmall cell lung cancer patients: More prone to development of bone and brain metastases? Lung cancer (Amsterdam, Netherlands). 2014;84(1):86-91.

[32] Tomasini P, Serdjebi C, Khobta N, Metellus P, Ouafik L, Nanni I, et al. Egfr and kras mutations predict the incidence and outcome of brain metastases in non-small cell lung cancer. International journal of molecular sciences. 2016;17:12.

[33] Weeraratna AT, Jiang Y, Hostetter G, Rosenblatt K, Duray P, Bittner M, et al. Wnt5a signaling directly affects cell motility and invasion of metastatic melanoma. Cancer cell. 2002;1(3):279-288.

[34] Kurayoshi M, Oue N, Yamamoto H, Kishida M, Inoue A, Asahara T, et al. Expression of wnt-5a is correlated with aggressiveness of gastric cancer by stimulating cell migration and invasion. Cancer research. 2006;66(21):10439-10448. 
[35] Liang H, Chen Q, Coles AH, Anderson SJ, Pihan G, Bradley A, et al. Wnt5a inhibits b cell proliferation and functions as a tumor suppressor in hematopoietic tissue. Cancer cell. 2003;4(5):349360.

[36] Bradner JE, Hnisz D, Young RA. Transcriptional addiction in cancer. Cell. 2017;168(4):629-643.

[37] Dittmer J. The role of the transcription factor ets 1 in carcinoma. Seminars in cancer biology. 2015;35(20-38):20-38.

[38] Xu TP, Wang YF, Xiong WL, Ma P, Wang WY, Chen WM, et al. E2f1 induces tincr transcriptional activity and accelerates gastric cancer progression via activation of tincr/stau1/cdkn2b signaling axis. Cell death \& disease. 2017;8(6):e2837.

[39] Lu Z, Luo RZ, Peng H, Huang M, Nishmoto A, Hunt KK, et al. E2f-hdac complexes negatively regulate the tumor suppressor gene arhi in breast cancer. Oncogene. 2006;25(2):230-239.

[40] Engelmann D, Mayoli-Nussle D, Mayrhofer C, Furst K, Alla V, Stoll A, et al. E2f1 promotes angiogenesis through the vegf-c/vegfr-3 axis in a feedback loop for cooperative induction of pdgf-b. Journal of molecular cell biology. 2013;5(6):391-403.

[41] Gorgoulis VG, Zacharatos P, Mariatos G, Kotsinas A, Bouda M, Kletsas D, et al. Transcription factor e2f-1 acts as a growth-promoting factor and is associated with adverse prognosis in non-small cell lung carcinomas. The Journal of pathology. 2002;198(2):142-156.

[42] Xiang J, Bandura J, Zhang P, Jin Y, Reuter H, Edgar BA. Egfr-dependent tor-independent endocycles support drosophila gut epithelial regeneration. Nature communications. 2017;8:15125.

[43] Hu B, Wang Q, Wang YA, Hua S, Sauve CG, Ong D, et al. Epigenetic activation of wnt5a drives glioblastoma stem cell differentiation and invasive growth. Cell. 2016;167(5):1281-1295 e1218.

[44] Sigismund S, Avanzato D, Lanzetti L. Emerging functions of the egfr in cancer. Mol Oncol 2018;12(1):3-20.

[45] Song X, Fan PD, Bantikassegn A, Guha U, Threadgill DW, Varmus H, et al. Erbb3-independent activation of the pi3k pathway in egfr-mutant lung adenocarcinomas. Cancer research. 2015;75(6):10351045.

[46] Truscott M, Islam AB, Lightfoot J, Lopez-Bigas N, Frolov MV. An intronic microrna links rb/e2f and egfr signaling. PLoS genetics. 2014;10(7):e1004493.

\section{Supplementary Figure Legends}

Figure S1. WNT5A suppresses EGFR-mutant NSCLC cells growth. a\&b The expression for WNT5A detected by qRT-PCR and western blotting in H1299 and HCC827 cell transfected with WNT5A or Mock. 
c\&d tumor weight at the end points of xenografts formed by subcutaneous injection of H1299 and HCC827 cells stably transfected with mock and WNT5A lentivirus into the dorsal flanks of nude mice $(\mathrm{n}=$ 4 for each group). ${ }^{\star} P<0.05$ vs. mock. ${ }^{*} P<0.01 P$ value was calculated by one-way ANOVA test.

Figure S2. E2F1 negatively regulated the expression of WNT5A. a Kaplan-Meier curves indicating the overall survival of NSCLC patients with different transcription factors expression, including AP-1 $(P<0.05)$, NF-kB $(P>0.05), P A X 2(P>0.05), P O U 2 F 1(P<0.05)$. log-rank test. $\mathbf{b}$ Analyze the correlation between the expression of AP-1 and WNT5A ( $R=0.06, P=0.429)$ (upper), POU2F1 and WNT5A $(R=-0.46, P<0.0001)$ (lower) using public datasets of tumor lung (NSCLC) (GSE33532) Pearson's product-moment correlation analysis. c Real-time qPCR showing the transcript levels of E2F1 (normalized to GAPDH) in H1975 and HCC827 cells stably transfected with E2F1 and sh-E2F1(\#1, \#2), compared with mock and sh-Scb (mean $\pm \mathrm{SD}, \mathrm{n}=5$ ). $\mathbf{d}$ Western blot showing the protein levels of E2F1 (normalized to GAPDH) in H1975 and HCC827 cells stably transfected with E2F1 and sh-E2F1(\#1, \#2), compared with mock and sh-Scb (mean $\pm \mathrm{SEM}, \mathrm{n}=5$ ). Student's $\mathrm{t}$ test analyzed the difference in $\mathrm{d} .{ }^{*} P<0.01 P$ value was calculated by one-way ANOVA test.

Figure S3 E2F1 inhibitor the WNT5A activity and facilitate downstream genes expression. a The two potential detail sequences of E2F1 binding to the promoter of WNT5A. b ChIP assay using E2F1 antibody indicating the endogenous binding of E2F1 to the promote of WNT5A in H1299 and HCC827 cells. The IgG antibody was taken as negative control and the $\mathrm{H} 3$ histone antibody was taken as positive control. C Dual-luciferase assays showing the promoter activity of WNT5A in 293T cells, and their changes in those stably transfected with mock, E2F1, sh-Scb, sh-E2F1 \#1 and sh-E2F1 \#2 (mean $\pm \mathrm{SEM}, \mathrm{n}=4$ ). Student's $\mathrm{t}$ test analyzed the difference in $\mathrm{d} .{ }^{*} P<0.05,{ }^{*} P<0.01, * \star * P<0.001$. p value was calculated by one-way ANOVA test. $\mathbf{d}$ The expression for $\beta$-catenin detected by western blotting in $\mathrm{H} 1299$ and HCC827 cell transfected wit WNT5A or Mock. e The expression of target genes was determined in $\mathrm{H} 1975$ and HCC827 cell with Ly294002 $(10.0 \mu \mathrm{M})$ by qRT-PCR. $\mathrm{f}$ TOP/FOP flash assay indicating the $\beta$-catenin activity in H1975 and HCC827 cells transfected with WNT5A and in those treated with solvent control (DMSO) or Ly294002 $(10.0 \mu \mathrm{M})$ for 24 hours. $\mathbf{g}$ The expression of indicated molecules was determined in $\mathrm{H} 1975$ and HCC827 cell treated with Ly294002 $(10.0 \mu \mathrm{M})$ for 48 hours by Western blotting. Data shown were the average of three independent experiments with similar results. The data are presented as the mean \pm SEM, $P$ values as determined by the t-test.

\section{Figures}


A

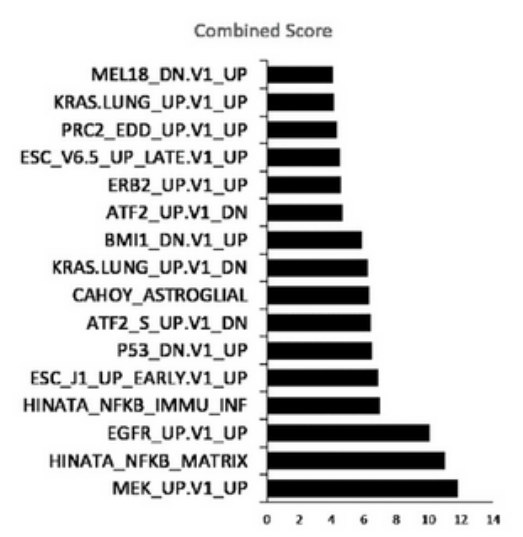

C

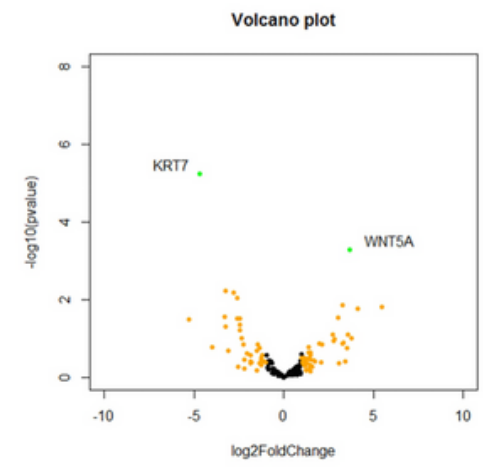

E

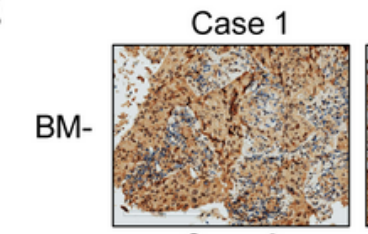

Case 3

$\mathrm{BM}+$

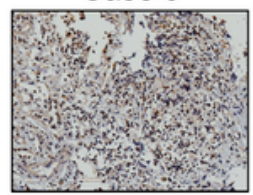

$\mathbf{F}$

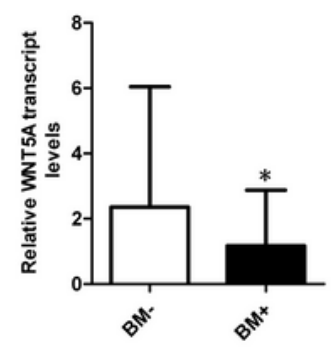

B

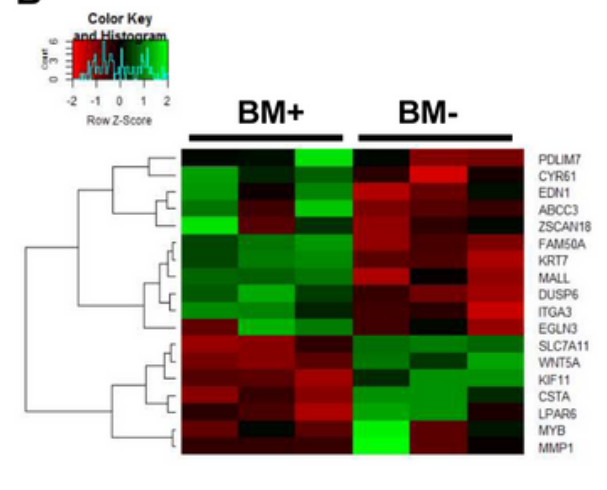

D
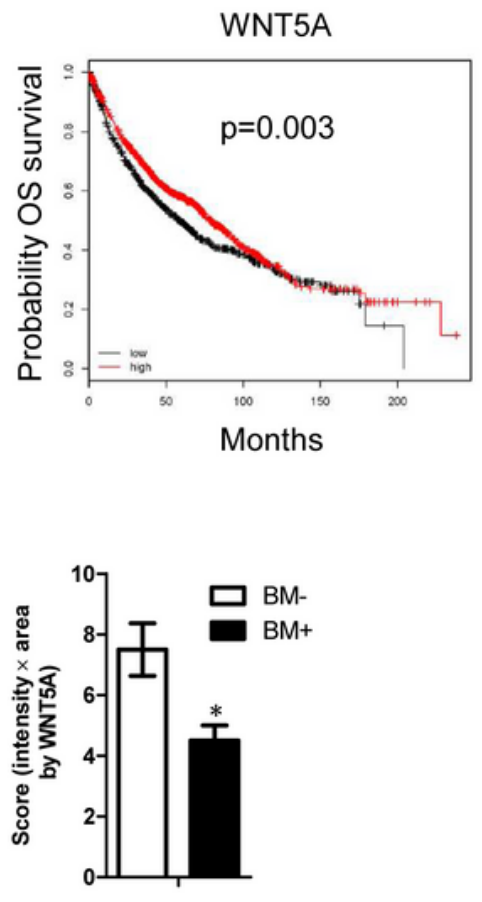

G

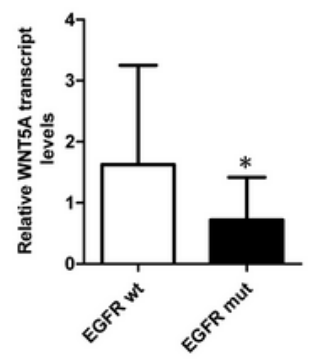

\section{Figure 1}

Identification of WNT5A associated with brain metastasis of NSCLC a MSigDB Oncogenic Signatures enriched by RNA sequencing data, 566 differentially-expressed genes identified and all oncogenic signatures with $p$-value less than 0.05 were indicated. b Heatmap for the differentially-expressed genes enriched in EGPR signature with BM+ or BM-. c Volcano plot for RNA profiling of 182 EGPR signature genes in RNA sequencing dataset, compared to BM+ group, according to the value of log2Foldchange 
$(\log 2 \mathrm{FC})$ and padj, Orange dots $(\log 2 \mathrm{FC}>1$, padj $>0.1)$, Green dots $(\log 2 \mathrm{FC}>1$, padj $<0.1)$, Black dots (log2FC $<1$, padj $>0.1)$ d Kaplan-Meier curves indicating the overall survival of NSCLC patients with high or low WNT5A expression (cutoff value $=368$, Affy ID:213425_at), log-rank test. e Representative IHC staining and quantitative analysis showing the expression of WNT5A in lung tumors from NSCLC patients with or without $B M$. ${ }^{*} P<0.05$, as determined by the t-test. $f$ qRT-PCR analysis of WNT5A expression in the plasma sample of BM- $(n=46)$ and BM+ NSCLC patients $(n=48)$ g qRT-PCR analysis of WNT5A expression in the 35 plasma sample of BM+ NSCLC patients with EGFR mutation $(n=25)$ or not $(n=10) .{ }^{*} P<0.05$, as determined by the t-test. 
A

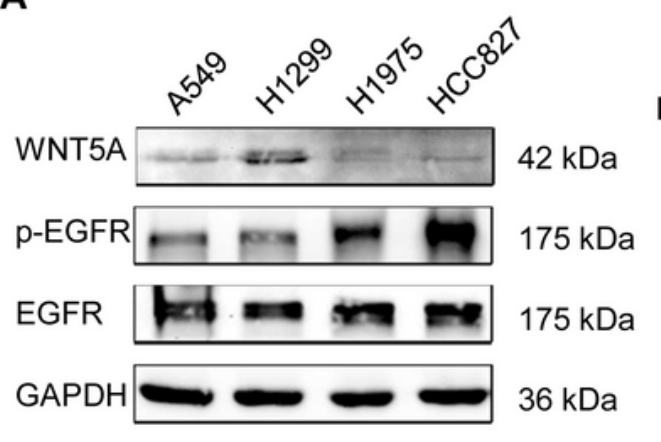

C
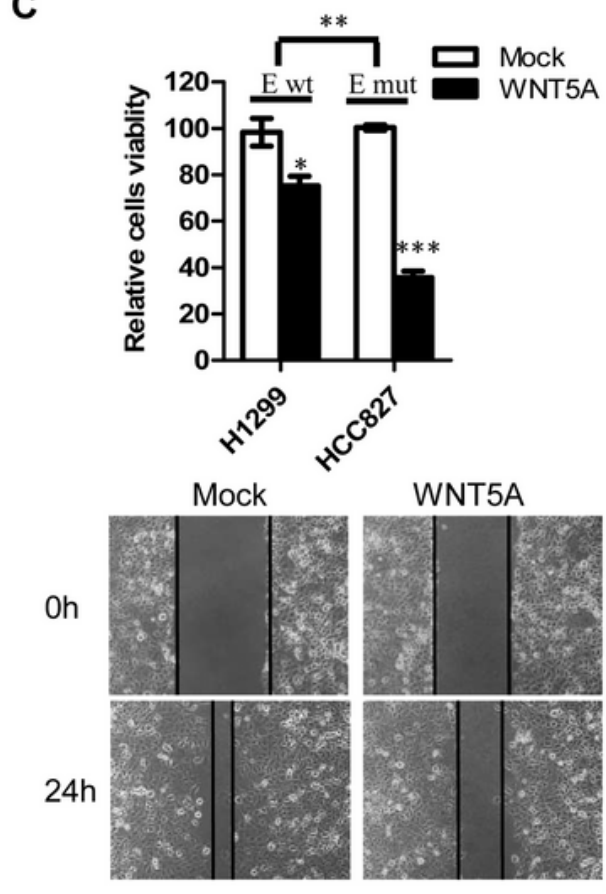

WNT5A

$\mathbf{E}$

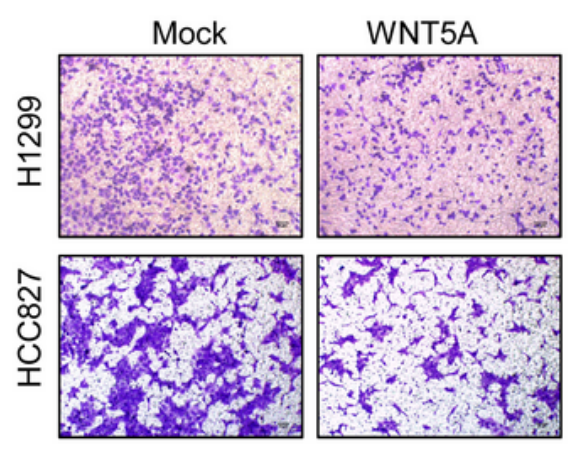

B

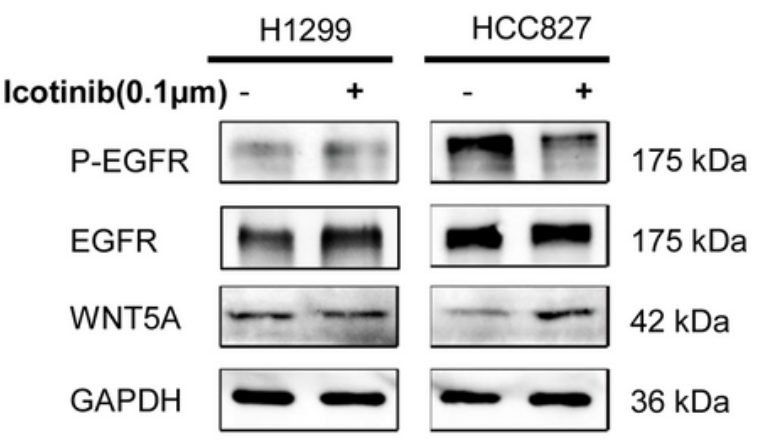

D
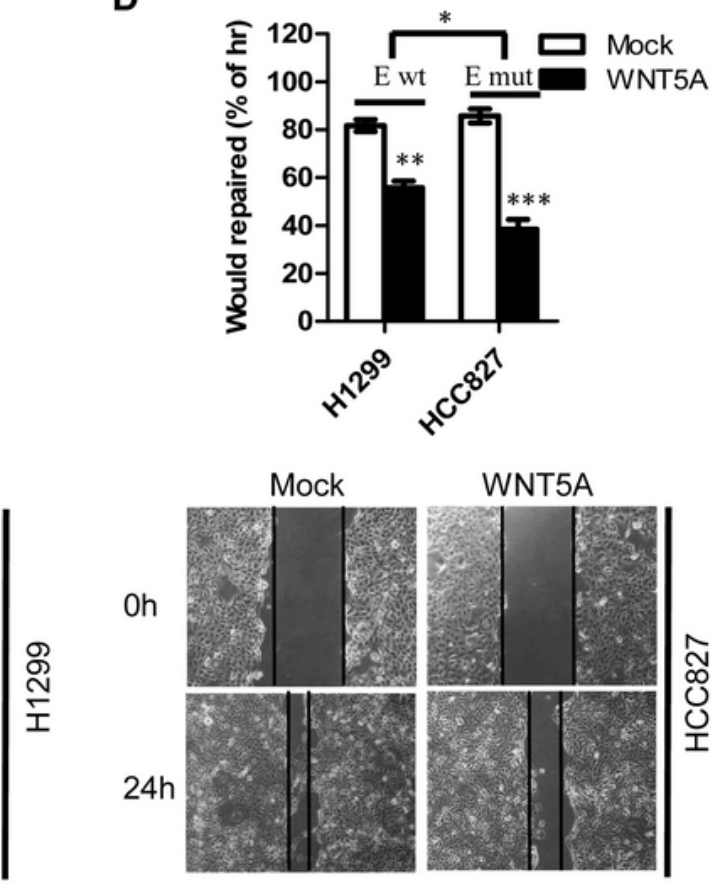

$\mathbf{F}$

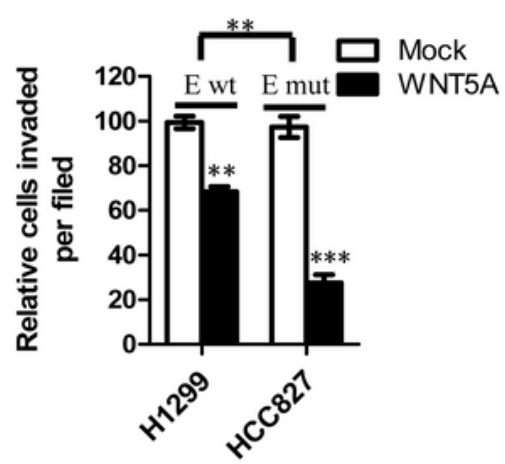

Figure 2

WNT5A expression was downregulated in EGFR mutated NSCLC cell lines. a The expression of WNT5A in NSCLC cell lines, including EGFR-mutant cell (H1975, HCC827) and EGFR-wt cell (A549, H1299) was detected by Western blotting. b cells were treated with icotinib $(0.1 \mu \mathrm{M})$ for 48 hours and proteins were evaluated for p-EGFR, total EGFR and WNT5A levels using Western blotting. c The proliferative ability of HCC827 and H1299 cells after transfection of WNT5A and vector (Mock) using CCK-8 assay. d Wound- 
healing assays were performed to assess NSCLC cells migration. Wound closure was determined $24 \mathrm{~h}$ after the scratch. e\&f Representative images and quantification of transwell assays indicated the invasive capability of NSCLC cells stably transfected with WNT5A and mock. bar $=50 \mu \mathrm{m}$. Data are expressed as mean $\pm \mathrm{SEM},{ }^{*} \mathrm{P}<0.05,{ }^{*} \mathrm{P}<0.01$, one-way ANOVA, unpaired two-sided $t$ test for two groups.

A

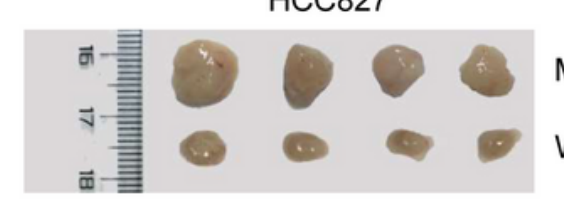

C

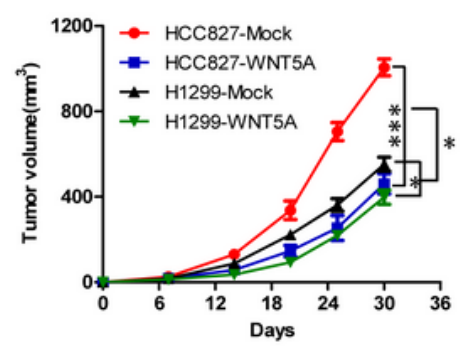

E
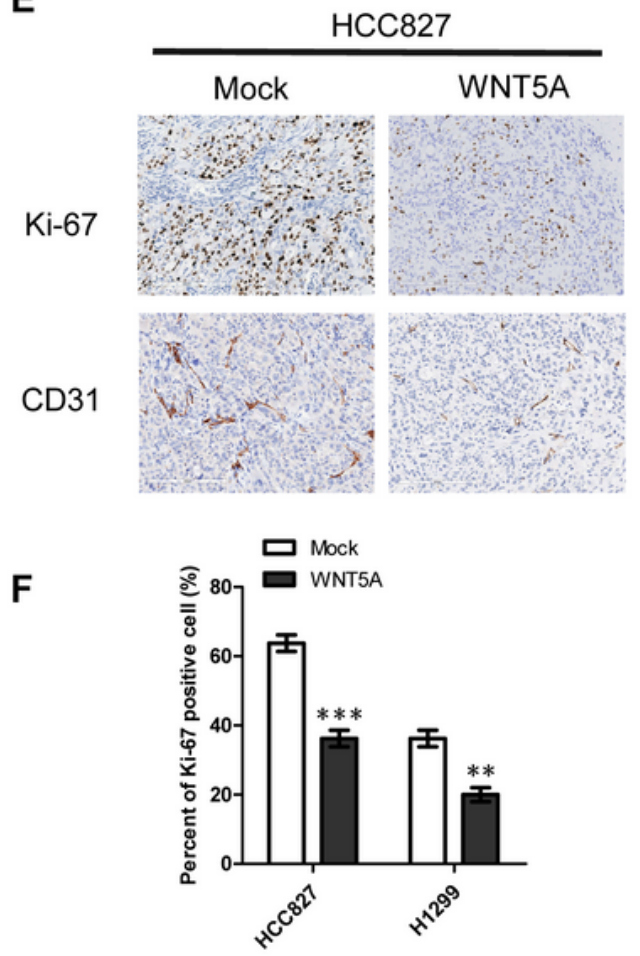

HCC 827
B

Mock

WNT5A

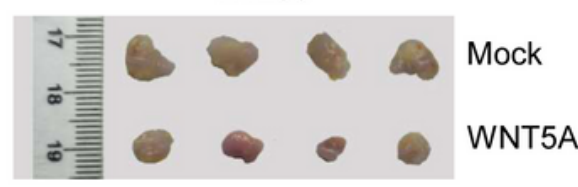

D

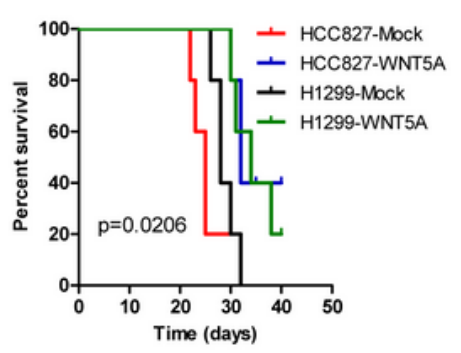

H1299
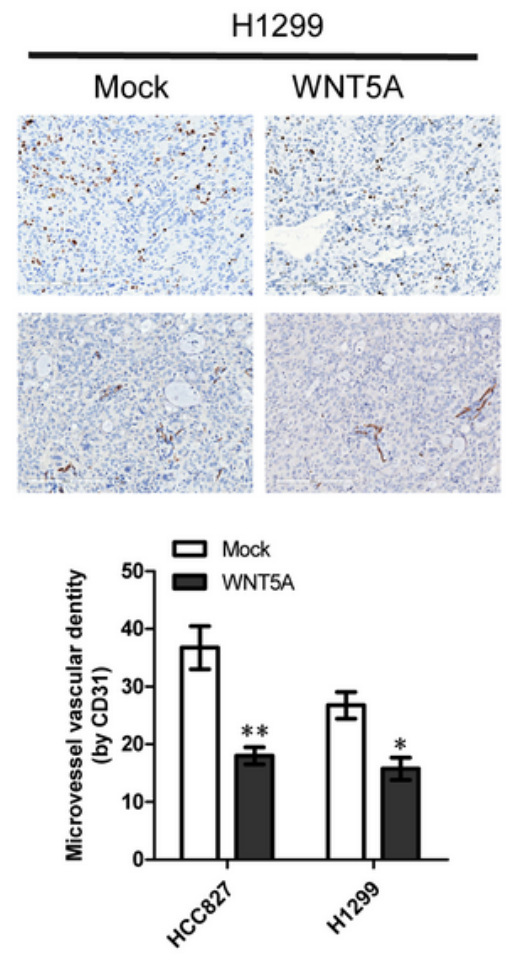

\section{Figure 3}


WNT5A inhibited the tumorigenesis in vivo. a\&b HCC827 and H1299 cells stably transfected with Mock and WNT5A were injected subcutaneously into nude mice $(n=4)$. Forty days after the injection, mice were photographed and killed. Tumor growth curves were plotted. c Compare differences in body weight data between the four groups $(P<0.05)$. $d$ Kaplan-Meier curves of nude mice treated with subcutaneous injection of HCC827 and H1299 cells stably transfected with Mock or WNT5A, ( $\mathrm{n}=5$ for each group). logrank test for survival comparison. e\&f Representative $\mathrm{IHC}$ staining and quantitative analysis of $\mathrm{Ki}-67$ and CD31 in xenograft tumors. Scale bars: $200 \mu \mathrm{m}$. The data are expressed as the mean $\pm S E M, * P<0.05$, $* * P$ $<0.01, * \star \star P<0.001$, as determined by unpaired two-sided $t$ test. 
A

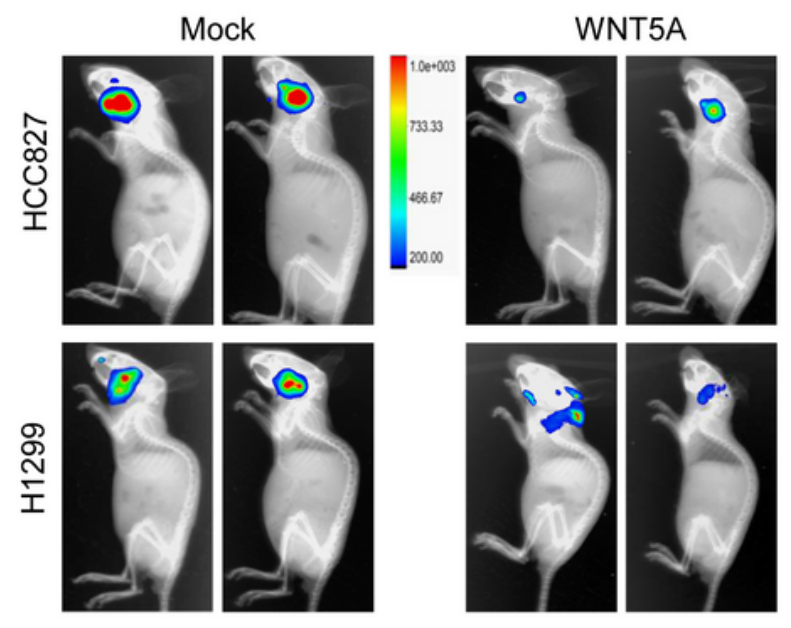

B

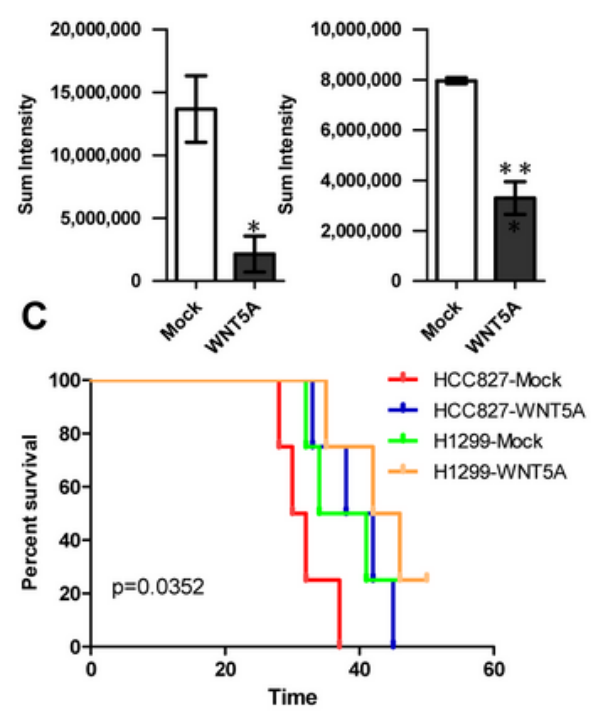

D
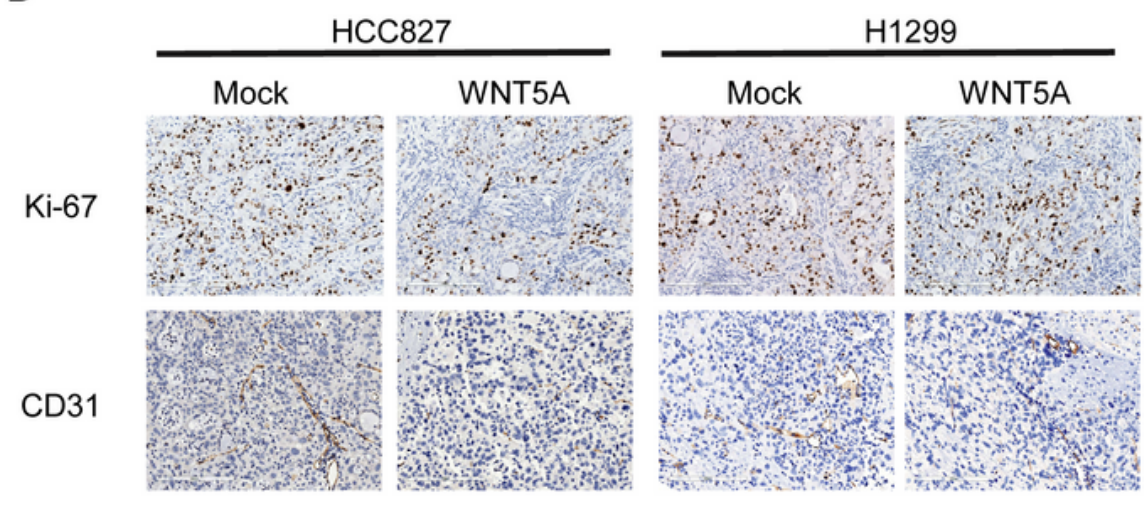

$E$

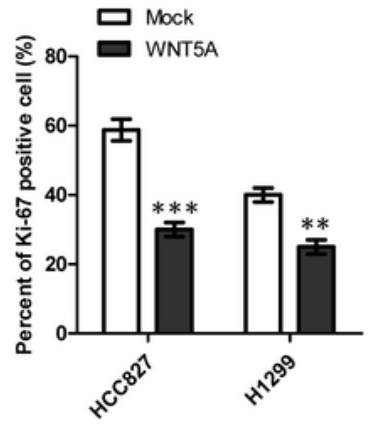

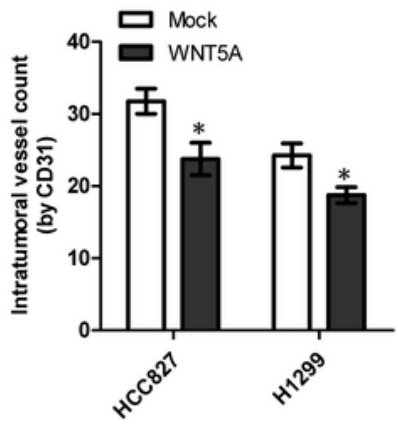

\section{Figure 4}

WNT5A inhibited the brain metastasis in vivo. a\&b Representative bioluminescent/photographic images in brain metastatic mice. $\mathrm{P}=0.0325$ in HCC827 group, $\mathrm{P}=0.01$ in $\mathrm{H} 1299$ group. $\mathrm{HCC} 827$ and $\mathrm{H} 1299$ cells stably transfected with Mock and WNT5A were injected into the intracarotid artery of nude mice $(n=5)$. *P $<0.05,{ }^{*} \mathrm{P}<0.01$, as determined by unpaired two-sided $t$ test. c Kaplan-Meier curves of nude mice treated with tail vein injection of HCC827 and H1299 cells stably transfected with mock or WNT5A ( $n=5$ 
for each group). Log-rank test for survival comparison. d\&e Representative IHC staining and quantitative analysis of Ki-67 and CD31 in brain xenograft tumors. Scale bars: $200 \mu \mathrm{m}$. The data are expressed as the mean $\pm S E M,{ }^{*}<<0.05,{ }^{\star} * P<0.01,{ }^{\star} * * P<0.001$, as determined by unpaired two-sided $t$ test.

A

D
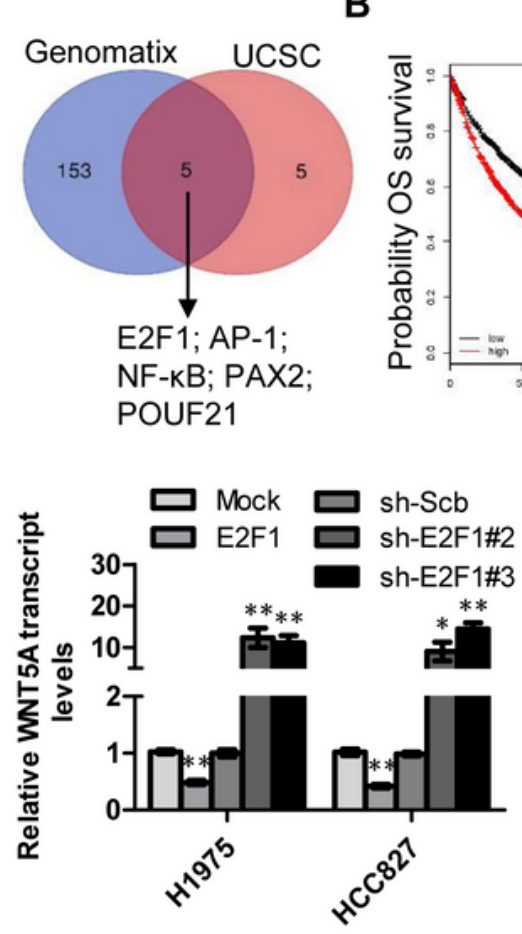

C

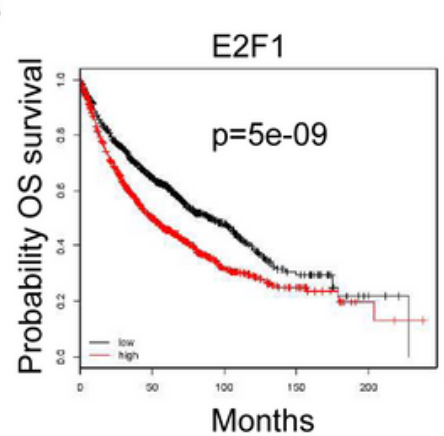

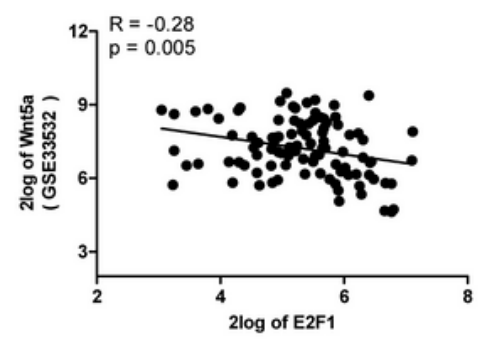

E

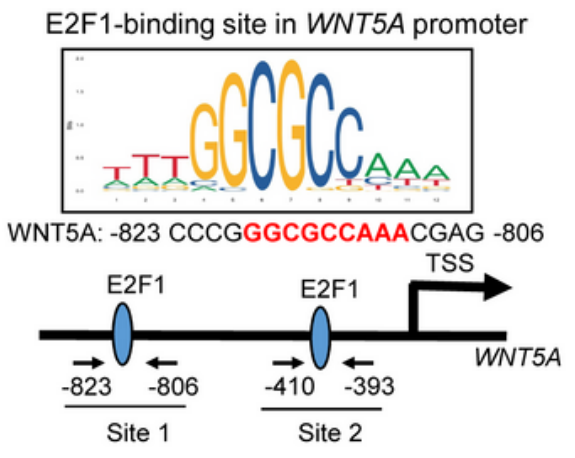

$\mathbf{F}$

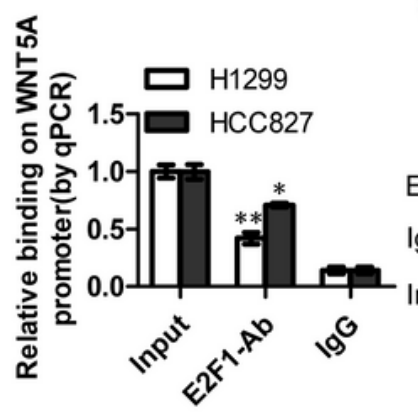

G
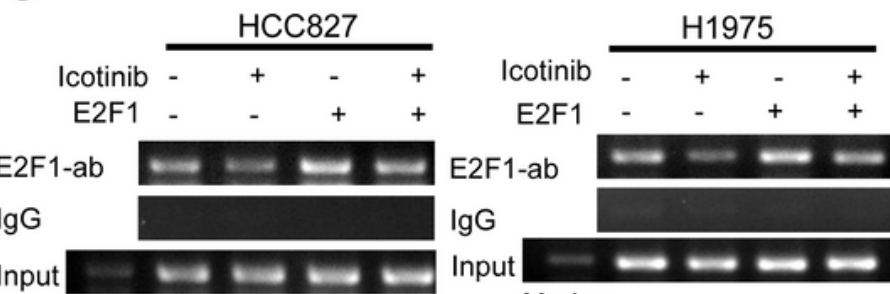

Marker

H

I
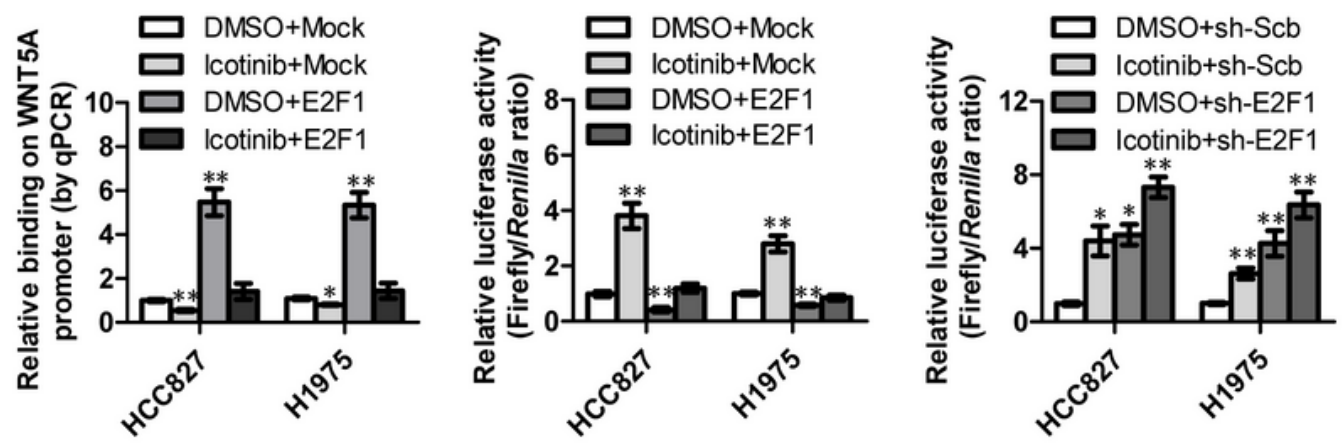

Figure 5

E2F1 was identified as transcription factor bind to the WNT5A promoter. a Venn diagram revealing the identification of transcription factors using public datasets of UCSC and Genomatix database. b Kaplan- 
Meier curves indicating the overall survival of NSCLC patients with high or low E2F1 expression. Log-rank test. c The correlation of E2F1 and WNT5A expression from GSE33532 database. Pearson's productmoment correlation analysis. $d$ The expression of WNT5A in H1975 and HCC827 cell by transfected E2F1 overexpression or knockdown vector and determined by qRT-PCR. e JASPAR analysis the core motif and detail sequence of E2F1 binding on the WNT5A promoter. $f$ ChIP-qPCR analysis of E2F1 binding on the WNT5A promoter in $\mathrm{H} 1299$ and $\mathrm{HCC} 827$ cell by use of E2F1 antibody or rabbit immunoglobulin $\mathrm{G}$ as control. g\&h ChIP and qPCR assay showed the enrichment level of E2F1 on WNT5A promoter with treatment of icotinib $(0.1 \mu \mathrm{M})$ and overexpression of E2F1 in EGFR-mutant cell. i WNT5A-luciferase reporter was transfected in $\mathrm{H} 1975$ and $\mathrm{HCC} 827$ cell with or without icotinib $(0.1 \mu \mathrm{M})$ and overexpression or knockdown of E2F1. Data shown were the average of three independent experiments. ${ }^{\star} \mathrm{P}<0.05$, ${ }^{\star *} \mathrm{P}<$ 0.01 . Student's $t$ test analyzed the difference. Differences among multiple groups were determined by one-way ANOVA with post-hoc Tukey HSD test. 
A

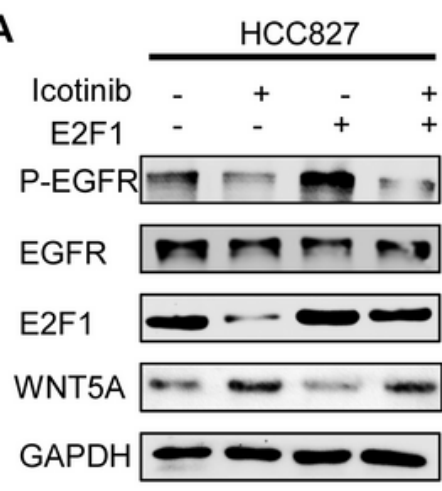

C

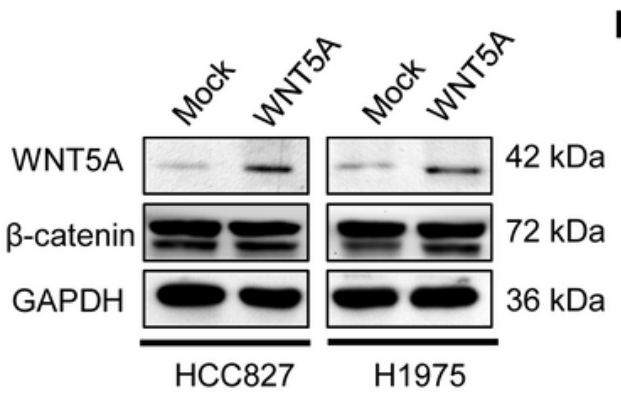

E

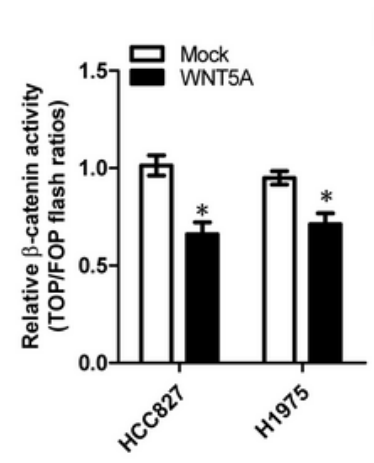

H

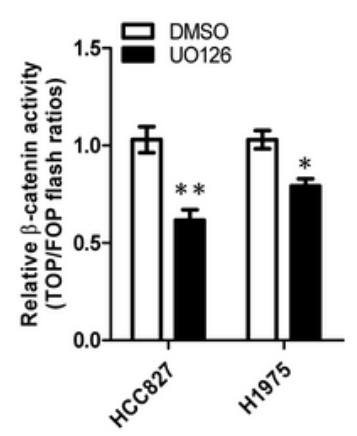

B

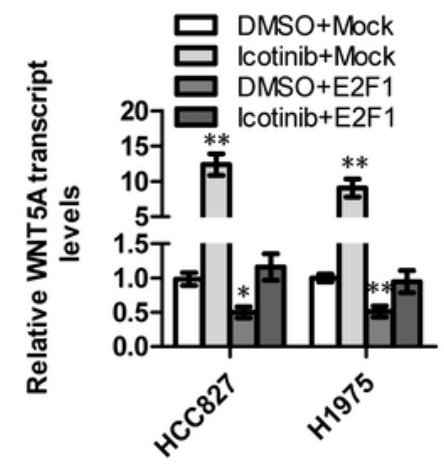

D
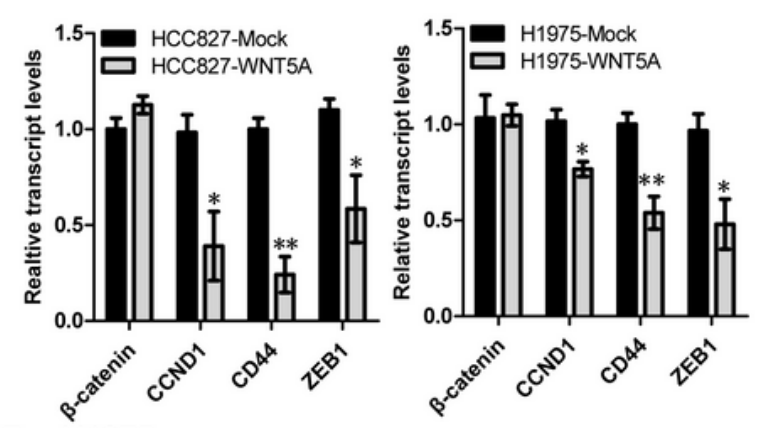

G

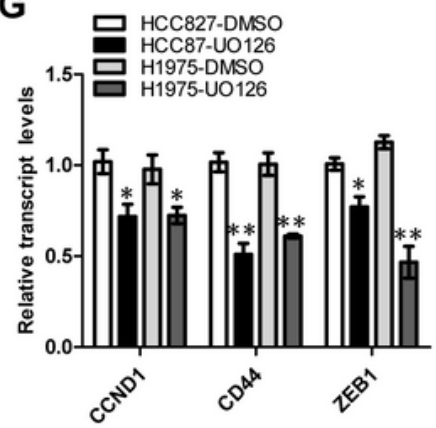

\section{I}

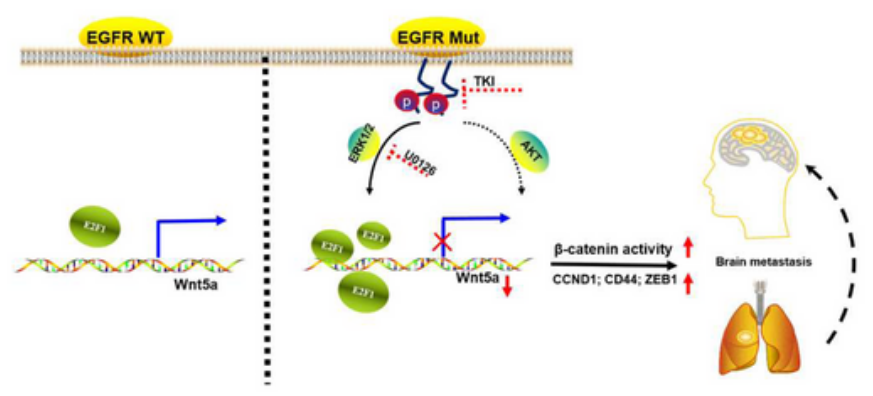

\section{Figure 6}

WNT5A regulated $\beta$-catenin activity and downstream genes expression dependent of ERK $1 / 2$ pathway in EGFR mutated cells. a\&b The expression of indicated molecules was determined in H1975 and HCC827 cell with or without icotinib $(0.1 \mu \mathrm{M})$ and E2F1 expression by qRT-PCR and Western blotting. c The expression of $\beta$-catenin in $\mathrm{H} 1975$ and HCC827 cell by Western blotting with or without transfected with WNT5A. d The expression of $\beta$-catenin and downstream genes CCDN1, CD44 and ZEB1 in H1975 and 
HCC827 cell by qRT-PCR with or without transfected with WNT5A. ${ }^{*} \mathrm{P}<0.05,{ }^{*} \mathrm{P}<0.01$, determined by unpaired two-sided $t$ test. e TOP/FOP flash assay indicating the $\beta$-catenin activity in $\mathrm{H} 1975$ and $\mathrm{HCC} 827$ cells transfected with WNT5A or vector (Mock). ${ }^{*} P<0.05$, as determined by unpaired two-sided $t$ test. $f$ The expression of indicated molecules was determined in H1975 and HCC827 cell with U0126ه10.0 $\mu \mathrm{M} \nabla$ by Western blotting. $\mathrm{g}$ The expression of target genes was determined in $\mathrm{H} 1975$ and HCC827 cell with U0126ه10.0 $\mu \mathrm{M} \varangle$ by $q R T-P C R$. $* P<0.05, * * P<0.01$, determined by unpaired two-sided $t$ test. $h$ TOP/FOP flash assay indicating the $\beta$-catenin activity in $\mathrm{H} 1975$ and $\mathrm{HCC} 827$ cells transfected with WNT5A and in those treated with solvent control (DMSO) or U0126 (10.0 $\mu \mathrm{M})$ for 24 hours. Data shown were the average of three independent experiments with similar results. ${ }^{*} \mathrm{P}<0.05,{ }^{*} \mathrm{P}<0.01$, determined by unpaired twosided $t$ test. The data are presented as the mean \pm SEM. i model depicting the role of EGFR, ERK1/2, E2F1 and WNT5A in EGFR-mutant lung cancer cells. E2F1 mediated WNT5A expression at transcriptional levels dependent on ERK1/2 pathway in EGFR mutated cells.

\section{Supplementary Files}

This is a list of supplementary files associated with this preprint. Click to download.

- figures1.tif

- figures2.tif

- figures3.tif

- SupplementaryFigurelegends.docx

- SupplementaryMaterial.pdf

- Tables3.xls

- SupplementaryMaterial.pdf

- figures3.tif

- figures2.tif

- figures1.tif

- Tables3.xls

- SupplementaryFigurelegends.docx 